\title{
ULTRAHIGH-BANDWIDTH VECTOR NETWORK ANALYZER BASED ON EXTERNAL ELECTRO-OPTIC SAMPLING
}

\author{
M. Y. Frankel, J. F. Whitaker, G. A. Mourou and J. A. Valdmanis \\ Ultrafast Science Laboratory, Department of Electrical Engineering and Computer Science, \\ University of Michigan, Ann Arbor, MI 48109-2099, U.S.A.
}

(Received 31 July 1991)

\begin{abstract}
We report the development of an ultrahigh-bandwidth vector network analyzer useful for small-signal characterization of high-speed semiconductor devices. It employs $100-\mathrm{fs}$ optical pulses for making terahertz-bandwidth electro-optic measurements of electrical signals, as well as for sub-picosecond, photoconductive, electrical-stimulus-signal generation. High-bandwidth coplanar strip transmission lines are used for signal transmission. A $0.15 \times 50 \mu \mathrm{m}$ gate $\mathrm{AlGaAs} / \mathrm{InGaAs} / \mathrm{GaAs}$ HFET has been characterized over a bandwidth of $100 \mathrm{GHz}$ using this network analyzer. A comparison with conventional RF network analyzer measurements performed to $40 \mathrm{GHz}$ demonstrated good agreement throughout this bandwidth. Such measurements of the actual device characteristics across their entire operating frequency range should improve device development and incorporation into active circuits.
\end{abstract}

\section{NOTATION}

$\alpha(f)$ frequency-dependent attenuation factor

$\beta(f)$ frequency-dependent phase factor

$d$ substrate thickness

$\epsilon_{\text {sos }}$ sapphire relative permittivity

$\epsilon_{\text {sup }}$ effective superstrate relative permittivity including the effect of an external electro-optic probe

$f_{\max } \quad$ transistor maximum frequency of oscillation

$S$ coplanar strip transmission line spacing between conductors

$\sigma \quad$ coplanar strip transmission line conductor d.c. conductivity

$S_{11} \quad$ forward reflection scattering parameter

$S_{12} \quad$ reverse transmission scattering parameter

$S_{21} \quad$ forward transmission scattering parameter

$t \quad$ coplanar strip transmission line conductor thickness

$V_{i} \quad$ actual incident electrical signal

$V_{\text {mi }}$ measured incident electrical signal

$V_{\mathrm{mr}}$ measured reflected electrical signal

$V_{\text {mt }} \quad$ measured transmitted electrical signal

$V_{\mathrm{r}}$ actual reflected electrical signal

$V_{t} \quad$ actual transmitted electrical signal

$W \quad$ coplanar strip transmission line conductor width

$z \quad$ electrical signal propagation distance

$Z_{\text {EEP }}$ perturbed transmission line characteristic impedance

$Z_{0}$ unperturbed transmission line characteristic impedance

\section{INTRODUCTION}

The continuing advances in the development of modern semiconductor devices have pushed the response of such devices to frequencies in excess of $400 \mathrm{GHz}[1]$. Frequency-domain characterization across this entire bandwidth range would be one requirement for facilitating further improvements and applications for devices. However, conventional, purely electronic measurement instruments such as vector network analyzers, spectrum analyzers and sampling oscillo- scopes are not effective at such high frequencies, the major limitation being imposed by the connectors and waveguides needed for signal coupling between the instrument and the device under test (DUT). This lack of convenient and accurate high-bandwidth device characterization methods imposes a serious obstacle to progress in semiconductor device development and utilization.

The recent development of ultrafast optoelectronic techniques has allowed novel high-bandwidth device characterization to be implemented[2-7]. These techniques rely on the unrivaled high-speed electrical signal generation and measurement capabilities provided by ultrashort optical pulses- $50 \mathrm{fs}-10$.ps duration emitted by various lasers. The photoconductive switching method[8] is used to generate picosecond-duration electrical stimulus signals which have bandwidths that extend for hundreds of gigahertz. For electrical measurements over similar bandwidths, either photoconductive[8] or electro-optic[9] sampling may be used, where the ultimate response of such techniques is limited by the duration of the photoconductively generated electrical stimulus signal, the bandwidth of the interconnecting transmission lines carrying the signals to be measured and the duration of the optoelectronic sampling gate.

There are two significant advantages that optoelectronic techniques possess over conventional, purely electronic ones. First, optoelectronic electrical signal generation and measurement bandwidths far exceed those achievable electronically. Secondly, both the stimulus-generation site and response-measurement planes are physically close to the DUT, so that it is possible to completely eliminate any interconnection discontinuities (transitions), as well as the 
errors and uncertainties introduced by the device de-embedding procedures. Optoelectronic techniques have been applied and demonstrated up to $100 \mathrm{GHz}$, and the measurement instruments based on these techniques should provide a viable complement to electronic ones.

In this paper we describe the application of the external electro-optic sampling (EEOS) technique[9] for the extraction of small-signal S-parameters of an active device. When combined with a high-bandwidth, coplanar-strip-transmission-line (CPS) test fixture[10], this technique allows one to determine device small-signal, frequency-domain characteristics over a range of $100 \mathrm{GHz}$, although it may also be extended to significantly higher frequencies. In Section 2, we discuss the parameters and the results affecting the selection of electrical signal generation, measurement and propagation methods. Based on these results, we have developed a complete smallsignal device characterization procedure. In Section 3, we demonstrate the implementation of this procedure to obtain device time-domain and frequency-domain characteristics.

\section{OPTOELECTRONIC SYSTEM}

Figure 1 shows the overall measurement system schematic. The optical pulse source is a balanced colliding-pulse mode-locked (CPM) laser which provides two 100 -fs pulse-trains at a $100 \mathrm{MHz}$ repetition rate and a wavelength of $620 \mathrm{~nm}[11]$. Since the optical pulse duration places a fundamental limit on the achievable measurement-system bandwidth, and the
CPM laser is currently the best source of ultrashort pulses in the visible region of the spectrum, this laser has been used in this investigation. The first beam is acousto-optically modulated at $3.5 \mathrm{MHz}$ and used to periodically excite a photoconductive switch. The second beam goes through the external electro-optic probe (Terametrics Model 200-TIR transducer) to gate the electric-field-induced birefringence which creates a polarization change in the probe[9]. The beam polarization change is converted via a Wollaston-prism analyzer to an intensity change detectable by the photodiodes. A compensator introduces additional birefringence to bias the intensity transmission function of this modulator to its $50 \%$ point, thereby making the intensity change linearly proportional to the local microwave electric field strength in the probe. The electro-optic probe and probing beam can be positioned to interrogate electrical signals appearing anywhere in the test fixture. A slowly varying time-delay is introduced between the excitation and the probing laser pulses by passing the probing beam through an optical delay rail; hence, a time-domain sampling scheme is realized.

A low-noise audio-frequency lock-in is used in conjunction with a front-end RF mixer to reduce the signal-to-noise threshold of the described system to sub-millivolt levels[12].

The photoconductor switch, used to generate electrical stimulus transients[8], consists of a gap within one of the metal transmission-line electrodes deposited on a semiconductor substrate. The switch gap typically has a very high dark resistance and is d.c. biased. The laser excitation pulse, with photon energy

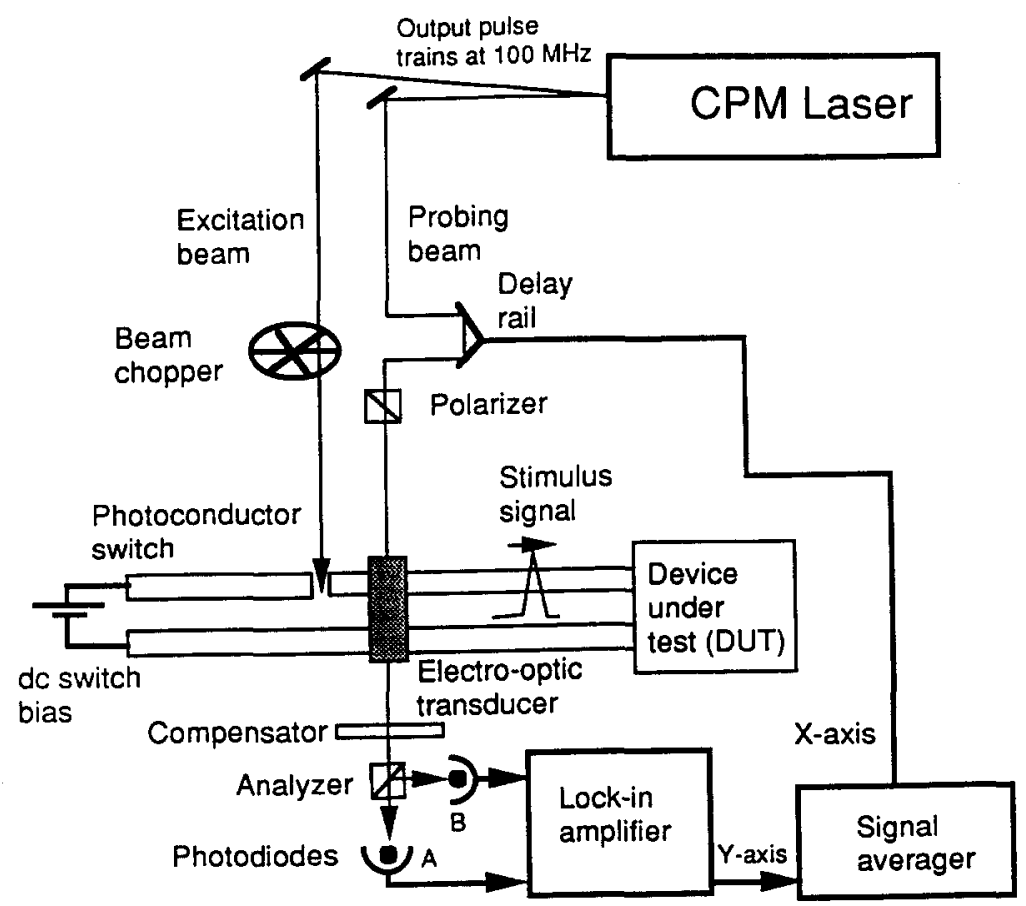

Fig. 1. Optoelectronic device characterization system schematic. 
above that of the bandgap of the photoconductor, illuminates the gap, is absorbed, and creates an electron-hole plasma, thereby electrically shorting the gap. Thus, an electrical transient signal which propagates along the transmission line is generated.

Three parameters affect the electrical bandwidth of this transient signal-electron-hole pair generation rate, electron-hole pair lifetime and switch $R C$ time constant. The generation of the electron-hole pairs in the semiconductor follows the incident optical pulse envelope to within the limitations imposed by the uncertainty principle, assuming the optical pulse spectrum to be within the semiconductor absorption band[13]. Hence, for 100-fs optical pulses, the electrical signal bandwidth is determined by the switch geometry and by the carrier lifetime in the semiconductor. Proper design of the photoconductor switch geometry to minimize its $R C$ time constant makes the carrier lifetime the limiting mechanism. So, the risetime of the generated electrical signal is determined by the optical pulse duration and the falltime by the carrier lifetime in the semiconductor. To maximize the electrical signal bandwidth, the transmission lines are fabricated on an ion-implant damaged Si-on-sapphire (SOS) substrate with a picosecond carrier lifetime[14]. Thus, the generated electrical signal has a single picosecond duration corresponding to a $\sim 35 \mathrm{GHz}$ bandwidth.

The external electro-optic sampling technique, because of its unsurpassed bandwidth, is used to measure the picosecond and subpicosecond electrical signals generated for device excitation in this work. Also, it does not require on-circuit, photoconductive sampling gate structures, and it is independent of the circuit substrate material. Figure 2 shows the measurement position of an external electro-optic probe (EEP) fabricated from an $\mathrm{Li}$ tantalate $\left(\mathrm{LiTaO}_{3}\right)$ crystal relative to a signal-carrying transmission line and the optical probing team. The accuracy and invasiveness of such a measurement arrangement have been investigated[15], and it has been shown that a properly designed external probe allows accurate measurements to be made with terahertz bandwidth.

Several perturbations in a propagating electrical waveform can result from the interaction of the electrical signal with the probe tip. First, reflections at the points where the guided waveform enter and exit the EEP input and output discontinuities are linear and cancel out in the frequency domain, where only the relative difference in the signals detected at two different measurement points is typically desired. However, perturbations have to be accounted for in situations where their influence on signals being measured is not identical. This occurs when a signal which has traveled through the tip, reflected from a discontinuity farther down the line, and returned to the tip is being measured. The perturbation introduced by the EEP itself can be modeled as a small reduction of the transmission line characteristic impedance over the distance the footprint of the probe overlaps the transmission line. Although the relative dielectric permittivity of the $\mathrm{LiTaO}_{3}$ EEP material is $\sim 43$, the effective superstrate permittivity introduced by the EEP is substantially reduced to $\epsilon_{\text {sup }} \sim 5$ due to the unavoidable presence of a small air gap between the circuit substrate and the EEP. The $\epsilon_{\text {sup }}$ is even lower than the substrate relative permittivity, $\epsilon_{\text {sos }} \sim 10.4$ and thus its influence on the effective permittivity and the characteristic impedance of the CPS is small, although not negligible.

As mentioned previously, the electro-optic measurement is sensitive to the electric field. To obtain a voltage calibration, an electrical signal of $1.0 \mathrm{~V}$ is applied to the transmission line at the modulation frequency of $3.5 \mathrm{MHz}$. The induced birefringence of the electro-optic probe is detected and the resulting lock-in offset signal provides the calibration information necessary to determine any transient electrical

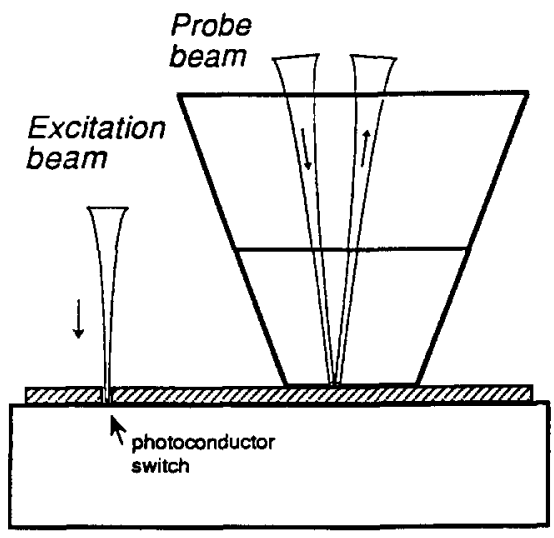

Side view

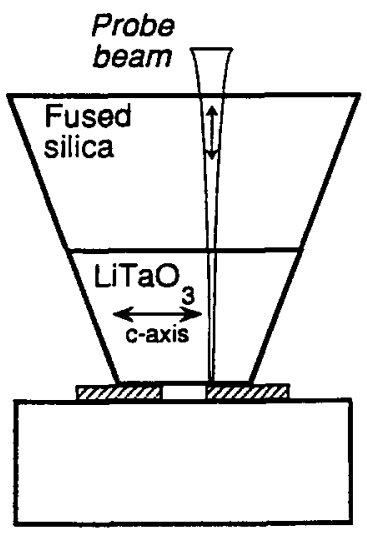

End view

Fig. 2. Schematic of the external electro-optic probe, the coplanar strip transmission line and the probing beam alignment. 
signal amplitude on the transmission line. Since the calibration signal is applied externally, care must be taken to determine its actual amplitude at the point where the short-pulse measurements are to be made.

The selection of microstrip or coplanar strip transmission line for the device test fixture is dictated by the considerations of high-bandwidth electricalsignal generation and propagation properties. The microstrip bandwidth is primarily determined by the thickness of the dielectric substrate, and mechanical limits on this dimension impose an upper boundary on its bandwidth. However, the CPS is photolithographically defined and can be scaled to much higher bandwidths.

Figure 3 shows the electrical waveforms generated by photoconductor switches embedded into $\sim 50 \Omega$ impedance microstrip ( $90 \mu \mathrm{m}$ wide top electrode, $100 \mu \mathrm{m}$ thick sapphire substrate) and $\sim 60 \Omega$ impedance CPS transmission lines (for dimensions see Section 3), as measured $\sim 300 \mu \mathrm{m}$ from the gap. The microstrip signal is longer in time indicating lower bandwidth. The electrical signal round-trip time between the top electrode and the ground plane ( $\sim 2 \mathrm{ps})$ is greater than the switch response time ( $\sim 1 \mathrm{ps}$ ), resulting in the substantial ringing evident in the microstrip signal. Thus, the CPS geometry was found to be a better choice for high-bandwidth electrical signal generation and propagation. Additionally, the planar nature of the CPS potentially allows for a parasitic-less integration with the planar devices under test (i.e. connections through via holes to the microstrip ground plane are not necessary).

The propagation properties of the CPS have been investigated with terahertz bandwidth to determine the geometry most suitable for the vector network analyzer application[10]. The obtained analytic formulas allow for an accurate and convenient computation of the CPS high-frequency attenuation $\alpha(f)$ and dispersion $\beta(f)$ characteristics.

The low-frequency propagation characteristics are equally important for a truly broadband characterization. In particular, the low-frequency dispersion and attenuation effects have been shown by Paulter et al.[16] to cause a propagating picosecond electrical pulse to develop a low-amplitude, trailing shoulder

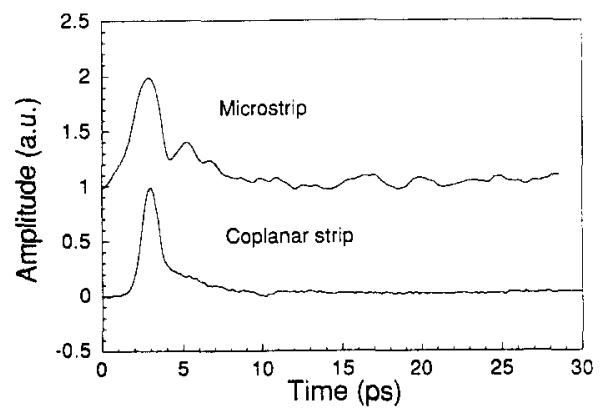

Fig. 3. Comparison of photoconductively generated electrical transients on microstrip and coplanar strip transmission lines.

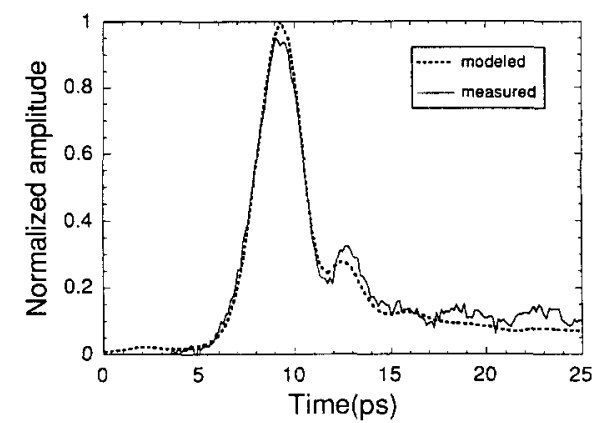

Fig. 4. Comparison of modeled and measured picosecond pulse propagation on a CPS with $S=5 \mu \mathrm{m}, W=50 \mu \mathrm{m}$, $t=1.2 \mu \mathrm{m}, \sigma=2 \times 10^{7} \mathrm{~S} \mathrm{~m}^{-1}, d=430 \mu \mathrm{m}, \epsilon_{\mathrm{sos}}=10.4$.

that may last for tens or even hundreds of picoseconds. To investigate this parasitic effect, a CPS analysis procedure has been implemented following the work of Gupta et al.[17] for a coplanar waveguide. To verify the accuracy of this procedure, we have measured and modeled picosecond electrical pulse propagation with the dimensions specified in Fig. 4. The excellent agreement between the measured and the modeled risetime, amplitude, falltime and trailing shoulder evident in this figure justifies the assumption that the model behaves adequately at both low- and high-frequency extremes.

The shoulder formation is attributed to the ohmic contribution of the conductor in the dispersion and the attenuation. Most of the geometric parameters of the CPS are fixed, as they are dictated by the requirements on characteristic impedance, minimum attenuation and dispersion, coupling to the device and physical realizability. The parameter that can be varied and that is shown to strongly influence the low-frequency CPS characteristics is the conductor thickness. Figure 5 shows the effects of $5 \mathrm{~mm}$ of CPS propagation on a 1 ps FWHM $1 \mathrm{~V}$ amplitude Gaussian pulse. The modeled pulses have been normalized to a unity peak amplitude which is off the scale of the graph to emphasize the shoulder formation (the actual peak amplitudes are indicated on the figure). The thinnest, $0.5 \mu \mathrm{m}$ conductor clearly shows a very

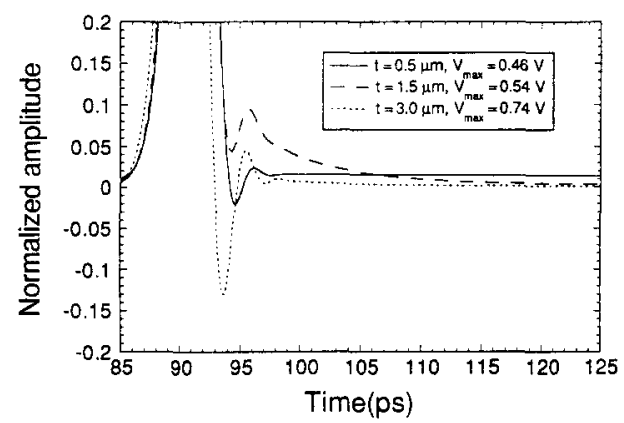

Fig. 5. Modeled 1 ps FWHM $1 \mathrm{~V}$ Gaussian pulse after $5 \mathrm{~mm}$ of propagation on a CPS with $S=5 \mu \mathrm{m}, W=50 \mu \mathrm{m}$, $\sigma=2 \times 10^{7} \mathrm{~S} \mathrm{~m}^{-1}, d=430 \mu \mathrm{m}, \epsilon_{\mathrm{sos}}=10.4$ and with thickness $t$ as parameter. 


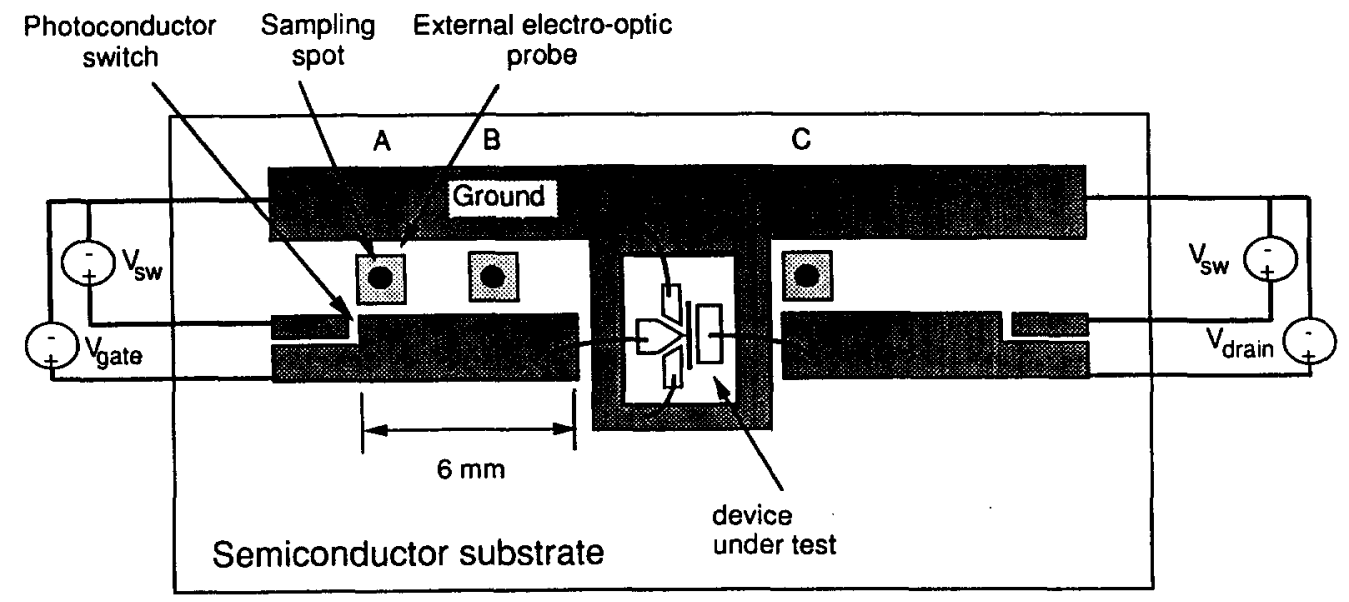

Fig. 6. Ultrahigh-bandwidth vector network analyzer device holder.

long, low-amplitude shoulder due to the high resistive attenuation and dispersion at low frequencies. The low-frequency dispersion is highest for the $1.5-\mu \mathrm{m}$ thick conductor and produces the highest initial shoulder amplitude. However, the shoulder decay time is also faster due to the reduced attenuation. Finally, although low-frequency dispersion is still present for the $3.0 \mu \mathrm{m}$ conductor, the reduced attenuation leads to the disappearance of the shoulder in preference of increased ringing caused by the dominance of high-frequency dispersion.

\section{DEVICE CHARACTERIZATION}

The results from the previous section were used to develop a CPS based vector network analyzer (VNA), as shown in Fig. 6. The symmetric design of the VNA allows a complete two-port characterization of a DUT wirebonded into this test fixture. The CPS had $50 \mu \mathrm{m}$ wide conductors separated by $5 \mu \mathrm{m}$, and it was fabricated on an ion-implant-damaged, SOS substrate, resulting in a characteristic impedance $Z_{0} \approx 60 \Omega$ (including finite metalization thickness effects).

As discussed previously, the optical excitation of a d.c.-based, ion-implanted SOS photoconductor switch results in the generation of picosecond electrical pulses. Photoconductor switches are embedded into the CPS and the d.c.-bias voltages are brought to the DUT along the same lines as the broadband electrical stimulus pulses.

Two spatially separated measurement points on the input CPS (A and B on Fig. 6) were used to experimentally obtain waveforms which were compared with each other to determine the broadband attenuation $\alpha(f)$ and dispersion $\beta(f)$ characteristics of the line. One measurement point on the input CPS (B on Fig. 6) was used to obtain both the incident and reflected gate signals. It was separated by $1.2 \mathrm{~mm}$ from the DUT to allow the incident signal to recover to its baseline before the reflected signal was detected. The incident and reflected pulses were thus not superimposed and a distinct time window was defined for each signal. Similarly, the $6 \mathrm{~mm}$ input CPS is sufficiently long so that one can separate in time the reflected DUT signal from signals arising at other discontinuities.

A measurement $200 \mu \mathrm{m}$ from the output of the DUT (C on Fig. 6) was used to obtain the forward transmission signal. Again, the CPS was long enough to time-window the transmitted DUT signal from reflections due to discontinuities further down the CPS.

After the forward time-domain signals have been measured, the switch bias is applied to the other end of the test fixture, and an identical procedure is followed to determine the time-domain characteristics while exciting the output port (device drain).

The DUT employed for this experiment was a General Electric $0.15 \times 50 \mu \mathrm{m}$ gate pseudomorphic heterojunction field-effect transistor (HFET), developed and described by Smith et al.[18]. Its design was different from conventional high-electron-mobility transistors in that a planar doped layer was incorporated into the strained channel. It improves the conduction channel carrier density, which more than compensates for any degradation in the mobility. An $\mathrm{AlGaAs} / \mathrm{GaAs}$ superlattice is used below the channel to improve the carrier confinement. This device was chosen for its high-speed, large breakdown voltages, and lack of detrimental low-frequency effects due to DX-centers in the AlGaAs buffer layer.

Figure 7 shows the signal measured on the DUT gate input and drain output transmission lines. It should be noted that the resistive shoulder effects described earlier have already been subtracted out for clarity. The distinct incident and reflected waveforms demonstrate the benefits of removing the measurement plane from the DUT, allowing the signals to be time-windowed. As can be seen, the input signal recovers to its baseline before the refiected signal arrives from the DUT. The 6-mm-long input CPS assumes that the DUT reflected response itself recovers to its baseline before any other discontinuity 


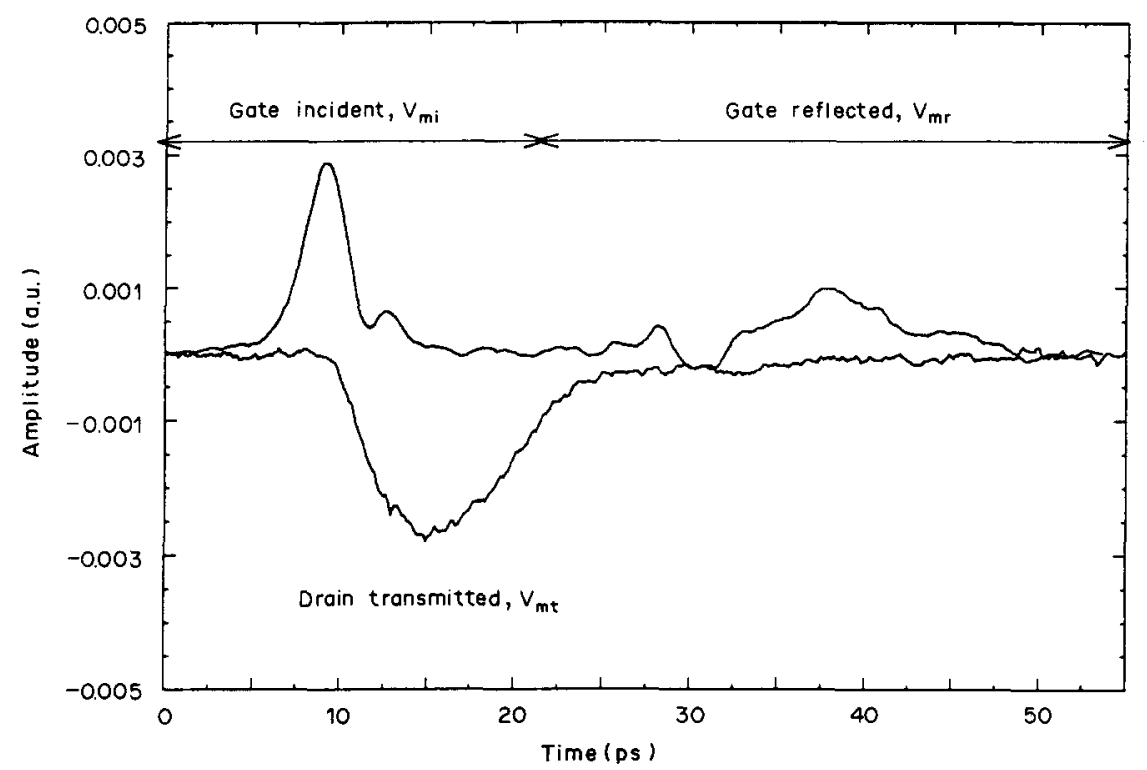

Fig. 7. Measured time-domain signals on the gate and drain transmission lines in the forward measurement mode.

reflections arrive at the measurement plane. Similarly, the drain output is seen to recover to the baseline completely within the measurement time-window.

The forward reflection scattering parameter (Sparameter) $S_{11}$ is computed from the ratio of the Fourier transform of the reflected and the incident time-domain signals. Since the EEP perturbs the signals slightly, as described in Section 2, we have to correct for its effects on the incident and reflected signals. As a first approximation, we take into account only single reflections at the EEP interfaces and ignore any resonant (multiple reflection) effects. Then, the ratio of the actual reflected and incident signals $V_{\mathrm{r}} / V_{\mathrm{i}}$ is given in terms of the measured ones $V_{\mathrm{mr}} / V_{\mathrm{mi}}$ as:

$$
\frac{V_{\mathrm{r}}}{V_{\mathrm{i}}}=\frac{Z_{0}}{Z_{\mathrm{EEP}}} \frac{V_{\mathrm{mr}}}{V_{\mathrm{mi}}},
$$

where the CPS characteristic impedance, perturbed due to the EEP over its footprint overlap, is given as:

$$
Z_{\mathrm{EEP}}=Z_{0} \sqrt{\frac{\epsilon_{\mathrm{sos}}+1}{\epsilon_{\mathrm{sos}}+\epsilon_{\mathrm{sup}}}} .
$$

Combining eqns (1) and (2), the gate reflection $\mathrm{S}$-parameter is computed as:

$$
S_{11}=\sqrt{\frac{\epsilon_{\mathrm{sos}}+\epsilon_{\mathrm{EEP}}}{\epsilon_{\mathrm{sos}}+1}} \frac{\mathrm{F}\left\{V_{\mathrm{mr}}\right\}}{\mathrm{F}\left\{V_{\mathrm{mi}}\right\}} \exp \{(\alpha+j \beta) z],
$$

where $\mathbf{F}\{\quad\}$ denotes a Fourier transform operation. The addition of the exponential term corrects for the small attenuation and dispersion introduced by the CPS and allows the effective measurement planes to be moved up to the device wirebonds.

However, the S-parameter obtained from eqn (3) is referenced to a $60 \Omega$ CPS transmission line used in the VNA. A straightforward impedance transformation is then employed to normalize this, and subsequent S-parameters, to a standard $50 \Omega$ impedance[19].

Additionally, at this time the absolute voltage level calibration of our electro-optic measurements is only accurate to $\sim 10-15 \%$. The VNA data has thus been scaled by a constant to exactly match the HP8510 data at low frequency $(2 \mathrm{GHz})$, where confidence is highest that the results should overlap. The VNA phase reference calibration can be obtained from the reflectance and transmission measurements of an unbiased (i.e. cold) transistor. This technique is analogous to the one in which a through-line is used as a reference standard. The advantage is that no actual through-line has to be wirebonded into the fixture, minimizing uncertainties due to unavoidable wirebond differences.

Figure 8a shows the comparison between the $S_{11}$ amplitude and phase obtained from the electro-optic measurements up to $100 \mathrm{GHz}$, with the conventional HP8510 network analyzer data acquired to $40 \mathrm{GHz}$.

The forward transmission scattering parameter $S_{21}$ is computed from the ratio of the Fourier transforms of the transmitted and the incident time-domain signals as:

$$
S_{21}=\frac{\mathrm{F}\left\{V_{\mathrm{mt}}\right\}}{\mathrm{F}\left\{V_{\mathrm{mi}}\right\}} \exp [(\alpha+j \beta) z] .
$$

No correction due to the EEP is necessary in this case since the transmitted signal is measured with the EEP removed from the input line and $V_{\mathrm{mt}} / V_{\mathrm{mi}}=V_{\mathrm{t}} / V_{\mathrm{i}}$. Figure 8b shows the $50 \Omega$ normalized $S_{21}$ amplitude and phase data from the electro-optic and conventional HP8510 network analyzer measurements.

Similarly, the reverse scattering parameters can be obtained from measurements at the other port. The reverse transmission parameter, $S_{12}$, in particular 


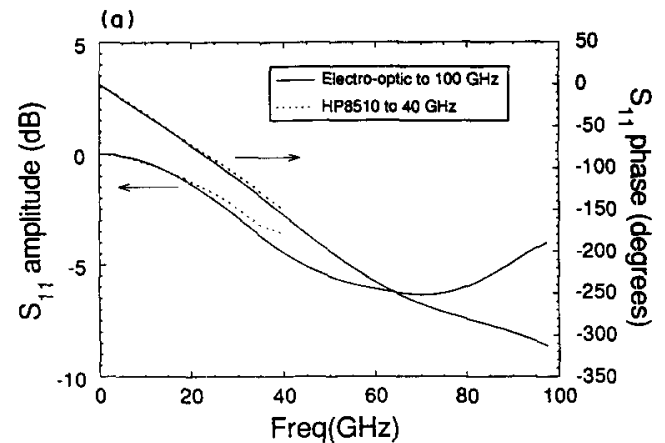

(b)

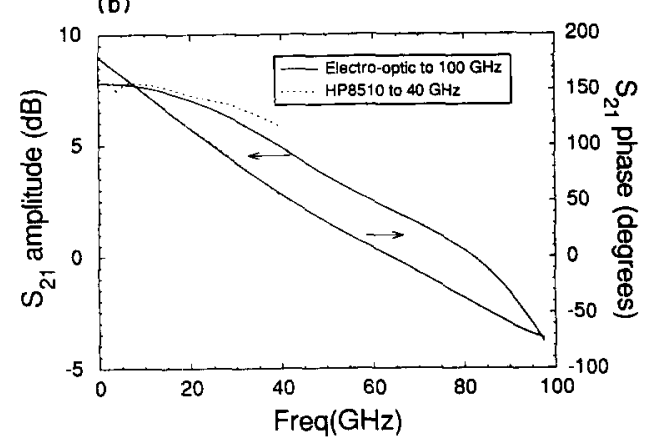

(c)

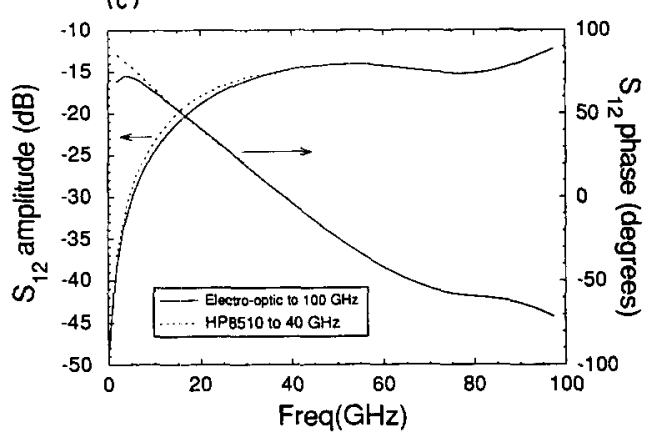

Fig. 8. S-Parameters measured electro-optically (up to $100 \mathrm{GHz}$ ) and with HP8510 (up to $40 \mathrm{GHz}$ ). (a) $S_{11}$; (b) $S_{21}$; (c) $S_{12}$

indicates the degree to which the transistor is unilateral. A good device would exhibit a very small reverse transmission, ideally tending to zero at d.c. and hence this parameter tests the limits of the dynamic range of the measurement system. This property for the electro-optic technique is indeed demonstrated by the reverse transmission $\left(S_{12}\right)$ measurements. Figure $8 \mathrm{c}$ shows the $50 \Omega$ normalized $S_{12}$ amplitude and phase data from the electro-optic VNA and HP8510 network analyzer. As can be seen, the match between the electro-optic measurement and the HP8510 measurement is good over the common frequency span within a range of $\sim 25 \mathrm{db}$. In general, the agreement between the two methods is good over a range of $\sim 40 \mathrm{db}$.

The disagreements can be attributed to the differences in the DUT wirebonds, which are not deembedded in either the RF or electro-optic measurements. Additional differences could be introduced by

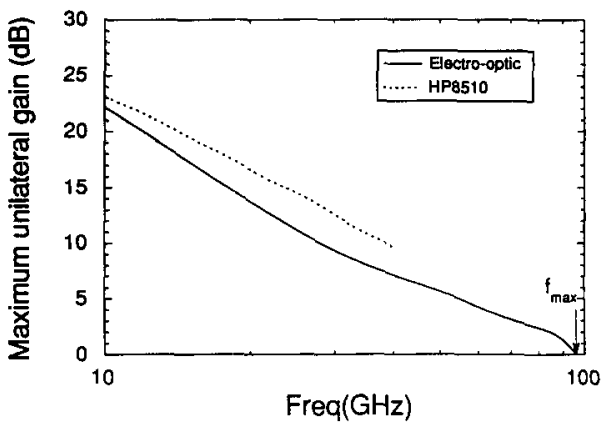

Fig. 9. Maximum unilateral gain computed from measured S-parameters.

the variation in the tested devices, which were not identical, but diced from the same wafer.

Conventional figures of merit, such as the unity current gain frequency and the maximum frequency of oscillation $\left(f_{\max }\right)$ can be determined from the measured S-parameters without need for extrapolation. Figure 9 shows the maximum unilateral gain curve computed from both the electro-optic and HP8510 measured S-parameters. The electro-optically, directly measured $f_{\max }$ is seen to be $\sim 94 \mathrm{GHz}$. This is slightly lower than that obtained from the extrapolated HP8510 measurements, although this could be due to the sources of error previously mentioned. Work is in progress to apply this technique to the characterization of much faster devices.

\section{CONCLUSION}

A vector network analyzer that utilizes photoconductor switches for picosecond electrical signal generation, the external electro-optic sampling technique for measurement, and coplanar strip interconnecting lines for electrical signal transmission has been developed and demonstrated. The vector network analyzer has been applied to the small-signal time and frequency-domain characterization of modern ultrafast transistors. A characterization bandwidth of $100 \mathrm{GHz}$ has been experimentally demonstrated (i.e. without need for extrapolation). Work is in progress to extend this bandwidth by several times, while characterizing devices with cutoff frequencies greater than those reported here.

Acknowledgements-M. Y. Frankel would like to thank Siemens, Munich for financial support. This work was supported by the Air Force Office of Scientific Research, University Research Initiative under Contract No. AFOSR90-0214.

\section{REFERENCES}

1. P. Ho, M. Y. Kao, P. C. Chao, K. H. G. Duh, J. M. Ballingall, S. T. Allen, A. J. Tessmer and P. M. Smith, Electron. Lett. 27, 325 (1991).

2. P. R. Smith, D. H. Auston and W. M. Augustyniak, Appl. Phys. Lett. 39, 731 (1981).

3. D. E. Cooper and S. C. Moss, IEEE J. Quant. Electron. QE-22, 94 (1986). 
4. M. Matloubian, H. Fetterman, M. Kim, A. Oki, J. Camou, S. Moss and D. Smith, IEEE Trans. Microwave Theory Tech. 38, 683 (1990).

5. C. Rauscher, IEEE Trans. Microwave Theory Tech. 39, 1179 (1991).

6. M. Y. Frankel, J. F. Whitaker, G. A. Mourou and J. A. Valdmanis, Picosecond Electronics and Optoelectronics Conf., Salt Lake City, UT (1991).

7. C. H. Lee, IEEE Trans. Microwave Theory Tech. 38, 596 (1990).

8. D.H. Auston, IEEE J. Quant. Electron. QE-19, 639 (1983).

9. J. A. Valdmanis, Electron. Lett. 23, 1308 (1987)

10. M. Y. Frankel, S. Gupta, J. A. Valdmanis and G. A. Mourou, IEEE Trans. Microwave Theory Tech. 39, 910 (1991).

11. J. A. Valdmanis and R. L. Fork, IEEE J. Quant. Electron. QE-22, 112 (1986).
12. J. M. Chwalek and D. R. Dykaar, Rev. Sci. Inst. 61, 1273 (1990).

13. P. P. Vasil'ev, Sov. J. Quant. Electron. 20, 209, (1990).

14. F. E. Doany, D. Grischkowsky and C.-C. Chi, Appl. Phys. Lett. 50, 460 (1987).

15. M. Y. Frankel, J. F. Whitaker, G. A. Mourou and J. A. Valdmanis, IEEE Microwave Guided Wave Lett. 1, 60 (1991).

16. N. G. Paulter, D. N. Sinha, A. J. Gibbs and W. R. Eisenstadt, IEEE Trans. Microwave Theory Tech. 37, 1612 (1989).

17. S. Gupta, J. F. Whitaker and G. A. Mourou, IEEE Microwave Guided Wave Lett. 1, 161 (1991).

18. P. M. Smith, L. F. Lester, P.-C. Chao, P. Ho, R. P. Smith, J. M. Ballingall and M.-Y Kao, IEEE Electron Dev. Lett. 10, 437 (1989).

19. G. Gonzalez, Microwave Transistor Amplifiers Analysis and Design. Prentice-Hall, Englewood Cliffs, NJ (1984). 